Western University

Scholarship@Western

Anatomy and Cell Biology Publications

Anatomy and Cell Biology Department

2012

\title{
Perceptual Enhancement of Arteriovenous Malformation in MRI Angiography Displays
}

\author{
Abhari Kamyar \\ Western University \\ John S. H. Baxter \\ Western University \\ Roy Eagleson \\ Western University \\ Terry Peters \\ Western University \\ Sandrine De Ribaupierre \\ Western University
}

Follow this and additional works at: https://ir.lib.uwo.ca/anatomypub

Part of the Anatomy Commons, and the Cell and Developmental Biology Commons

Citation of this paper:

Kamyar, Abhari; Baxter, John S. H.; Eagleson, Roy; Peters, Terry; and De Ribaupierre, Sandrine, "Perceptual Enhancement of Arteriovenous Malformation in MRI Angiography Displays" (2012). Anatomy and Cell Biology Publications. 60.

https://ir.lib.uwo.ca/anatomypub/60 


\section{Perceptual enhancement of arteriovenous malformation in MRI angiography displays}

Kamyar Abhari John S. H. Baxter Roy Eagleson Terry Peters Sandrine de Ribaupierre 


\title{
Perceptual Enhancement of Arteriovenous Malformation in MRI Angiography Displays
}

\author{
Kamyar Abhari ${ }^{a, b}$, John S.H. Baxter ${ }^{a}$, Roy Eagleson ${ }^{a, b, c}$, Terry Peters ${ }^{a, b, c}$, \\ and Sandrine de Ribaupierre ${ }^{c, d}$ \\ ${ }^{a}$ Imaging Research Laboratories, Robarts Research Institute; \\ ${ }^{b}$ Biomedical Engineering Program, The University of Western Ontario; \\ ${ }^{c}$ Department of Electrical and Computer Engineering, The University of Western Ontario; \\ ${ }^{d}$ Department of Clinical Neurological Sciences, The University of Western Ontario, London \\ Health Sciences Centre, London, Ontario, Canada
}

\begin{abstract}
The importance of presenting medical images in an intuitive and usable manner during a procedure is essential. However, most medical visualization interfaces, particularly those designed for minimally-invasive surgery, suffer from a number of issues as a consequence of disregarding the human perceptual, cognitive, and motor system's limitations. This matter is even more prominent when human visual system is overlooked during the design cycle. One example is the visualization of the neuro-vascular structures in MR angiography (MRA) images. This study investigates perceptual performance in the usability of a display to visualize blood vessels in MRA volumes using a contour enhancement technique. Our results show that when contours are enhanced, our participants, in general, can perform faster with higher level of accuracy when judging the connectivity of different vessels. One clinical outcome of such perceptual enhancement is improvement of spatial reasoning needed for planning complex neuro-vascular operations such as treating Arteriovenous Malformations (AVMs). The success of an AVM intervention greatly depends on fully understanding the anatomy of vascular structures. However, poor visualization of pre-operative MRA images makes the planning of such a treatment quite challenging.
\end{abstract}

Keywords: MR Angiography, Arteriovenous Malformation, Contour Enhancement, Cel-Shading

\section{INTRODUCTION}

\subsection{Visualization of MR Angiography:}

Image-Guided Intervention (IGI) platforms must be designed in such a way as to maximize the information content for the user. However, some current visualization techniques, particularly those designed for minimallyinvasive surgery often include conflicting visual cues, lack of depth perception, poor user interfaces, ambiguous visual scenes, obscured valuable information, and arbitrary coordinate frames that require additional cognitive effort directed at spatial reasoning. These flaws arise through neglect or underestimation of the constraints and limitations of the human perceptual, cognitive, and motor system. Among these factors, human visual perceptual issues in particular require consideration throughout the design cycle of the display environment. The human visual system is extremely sensitive, yet prone to error if mixed visual cues are presented. ${ }^{1}$ The contour around an object is an important cue used by the perceptual system to determine the shape or relative depth of such objects within a scene. However, our perception of a visual scene is heavily dependent on a number of other factors such as binocular disparity, light intensity and so on. Therefore, in a scene where these properties are impoverished, contours can be a very important visual cue. In a clinical context, the importance of contour perception is critical in cases where clinicians are required to trace objects within medical datasets to better understand the anatomy. One example of such a technique is the visualization of the vasculature of the brain in MR angiography (MRA) volume data. Although MRA images can be very beneficial for diagnosis or pre-operative planning purposes, proper visualization of the blood vessels can be compromised when the target area is visually cluttered. We

Medical Imaging 2012: Image Perception, Observer Performance, and Technology Assessment, edited by Craig K. Abbey, Claudia R. Mello-Thoms, Proc. of SPIE Vol. 8318, 831809

(C) 2012 SPIE · CCC code: $1605-7422 / 12 / \$ 18 \cdot$ doi: $10.1117 / 12.911687$

Proc. of SPIE Vol. 8318 831809-1 
believe that the provision of enhanced visual contours will facilitate the perception of shape, orientation, and the spatial relations between blood vessels, which will result in improved diagnosis and pre-operative planning. In this study, we investigate a mode of visualization which combines the use of volumetric transfer functions with volume rendering techniques to enhance the perception of contours and shapes.

\subsection{Arteriovenous Malformation}

Arteriovenous Malformations (AVMs) are vascular lesions where abnormal connection between arteries and veins results into direct drainage of blood flow from the arterial to the venous system. The center of the lesion is called nest or nidus, and is often visible in angiography images as a tangled mass of blood vessels. Although AVMs can develop in many different sites, 300,000 Americans are affected by intracranial or spinal AVMs and about $1 \%$ of the affected population will die annually as a direct result of this condition. ${ }^{2}$ Proper planning is necessary for successful treatment of the AVM, which would not be possible without understanding the functional vascular anatomy. In many cases, planning is performed by forming a mental image of the nidus using 2D pre-operative MRA and/or DSA images, segmented 3D images taken out of their context (reconstruction of the vessel alone, without the visualization of the neighboring brain). When a surgical option is chosen, in addition to the understanding of the vascular anatomy, a good understanding of where the lesion lies in the brain relative to eloquent areas is essential to identify the best surgical path, and to determine the point of entry on the skull. In addition, the differentiation between arteries and veins is important since, to avoid an intraoperative hemorrhage, the arteries must be clipped and cut before the veins. In addition to this mental challenge, the inability to concurrent visualize multiple brain structures is another drawback of conventional planning systems. In reality, surgeons rely heavily on neuronavigation systems to locate the target area and remove the nidus intra-operatively. However, the images used by the neuronavigation system are not easily modifiable by the user to change the transparency of other neighboring structures, or change the aspect of the vessels so that the veins and arteries may be more easily identified. While the vessels can be segmented, the result is often insufficient to be able to follow each vessels separately in the nidus to determine their nature and their exact localization. As stated in the previous section, improving visualization of the blood vessels, can aid neurosurgeons to better plan their surgery so that arteries can be approached first with certainty. This enhancement technique can be an asset for AVM pre-operative planning. In this study, an expert's qualitative feedback on the approach discussed in this paper to visualize AVM patient MRA data is provided.

\section{MATERIALS AND METHODS}

\subsection{Materials}

\subsubsection{MR Angiography}

A number of MR-based techniques can be used to create Magnetic Resonance Angiography (MRA) images where blood vessels are visibly distinct due to high contrast between the vascular lumen and its surrounding tissues. Unlike catheter and CT angiography, MRA techniques are non-invasive, involve no ionizing radiation and are capable of providing 3D datasets. Such images can be used for evaluating arteries for aneurysms, stenosis, or occlusion as well as planning surgical approaches where understanding the vascular architecture of a lesion is a prerequisite of successful intervention. One such application is AVMs where identifying the arterial branches and differentiating them from draining veins using MRA images is necessary. ${ }^{3}$ Visualization of MRA images, if performed by conventional means, requires mental transformation and high cognitive effort to locate and pinpoint a certain vessel from one slice to another, or to localize the 3D reconstruction in the brain. While it has been demonstrated that 3D visualization of MRA data is a convenient and reliable surgical planning tool (e.g. ${ }^{4}$ ), we believe that exploring 3D visualization of MRA volumes can be further enhanced by providing extra visual cues to improve the perception of the shape, orientation, and order of blood vessels. This improvement can be achieved by emphasizing the contour around vessels in MRA images. 


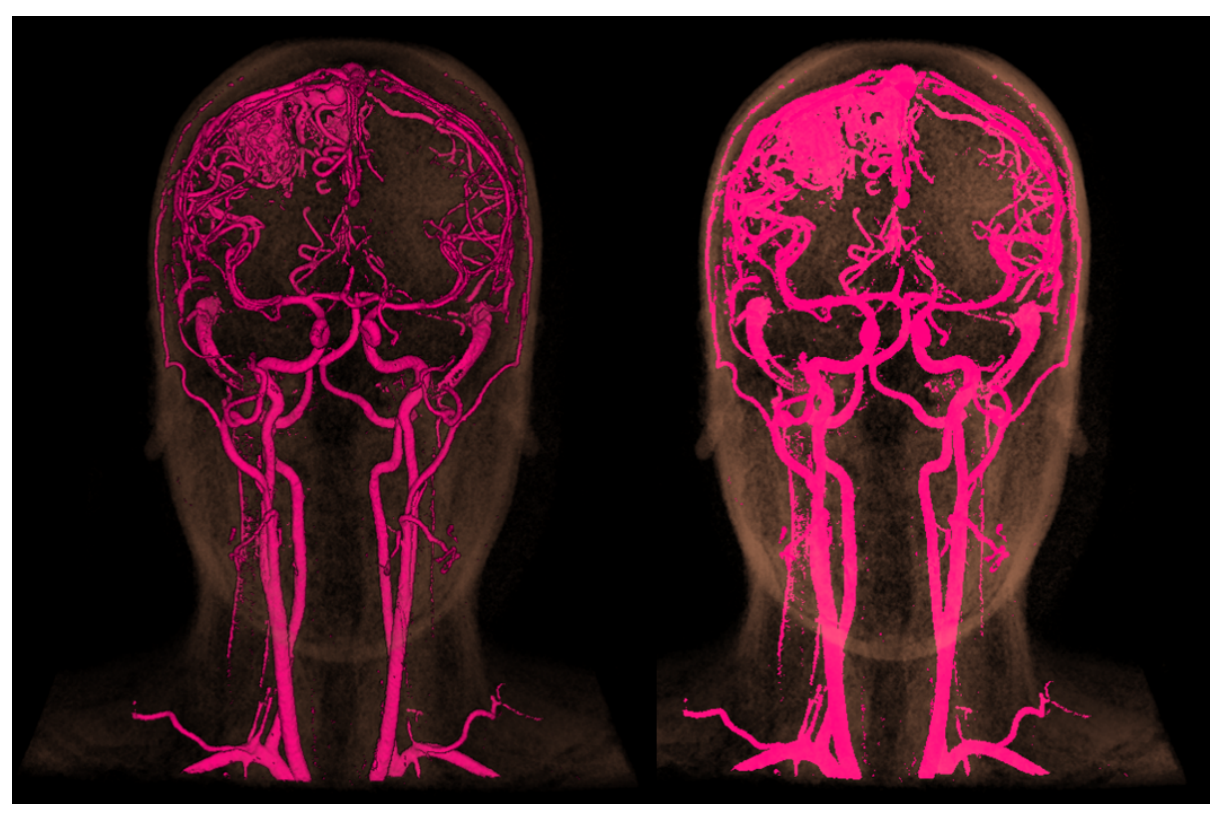

Figure 1. Contours are more salient in the left image using Cel-shading contour enhancement

\subsubsection{Direct Volume Rendering using 2D-Transfer Function (TF)}

Direct volume rendering (DVR $)^{5}$ is a technique used to visualize serially acquired 2D slices of 3D datasets in the form of a volume. In DVR, a Transfer Function (TF) assigns certain opacity and colour to every sample value by extracting features such as intensity (1D TF) or intensity and gradient (combined 2D TF). Thus, areas with similar characteristics (i.e. tissue type) can be represented with the same colour and opacity. The advantage of Volume Rendering (VR) over Surface-based Rendering is that, unlike the latter where a pixel either belongs to a certain tissue type or not (binary classification), in volume rendering techniques, voxels can represent mixed tissue interfaces (continuous classification, e.g. at the boundary of different materials). ${ }^{6}$ In this study, ray casting using a 2D TF was used to render $3 \mathrm{D}$ volume datasets. In this particular method, a number of rays are cast through the volume from the camera's viewpoint. While voxels that fall along the rays are sampled, these voxels are trilinearly interpolated since they are not necessary aligned with the rays. Interpolated sampled values are then shaded and coloured based on their normalized gradient vector, the source of light, and their opacity/colour values derived from the transfer function. Besides DVR, Maximum Intensity Projection (MIP) is also being used for visualizing blood vessels in angiographic images. In this method, voxels with the highest intensity that fall along rays cast from the camera's viewpoint are projected onto a $2 \mathrm{D}$ plane, ${ }^{7}$ resulting in a set of $2 \mathrm{D}$ images where blood vessels are visibly represented. One immediate disadvantage of MIP over VR is that the 3D relationships between vessels are not preserved due to its rendering process and lack of shading. ${ }^{6}$ For instance, in MIP images where veins and arteries are both depicted, the location of veins might appear further posterior than their actual anatomical location. ${ }^{6}$ Another drawback of MIP is that calcification may result in overestimating arterial stenosis. This is not a problem in volume rendering as the calcification and blood vessels are visualized individually. ${ }^{6}$ MIP has nevertheless been employed for more than two decades to visualize angiography images because of its simplicity and its fast processing speed. However, improvements in computer hardware technology and GPU programming during past few years have made it possible to visualize 3D datasets in real-time using VR techniques. ${ }^{8}$ Another advantage of MIP over VR is a better depiction of small intraparenchymal branches and less intra-observer variability. ${ }^{6}$ In this study, our main goal is to investigate the effect of contour enhancement on perceiving the spatial relations between vascular structures. Hence, VR is the method of choice due to its strength in representation of 3D relationship between different anatomies. We believe that VR visualization along with contour enhancement can be beneficial for those neuroclinical applications where understanding the 
relative relationship among intracranial vessels (e.g. AVM, Vein of Galen malformation) or between vessels and other brain structures (e.g. vascular tumour) is necessary.

\subsubsection{Contour perception}

Perceiving an object distinct from its surrounding environment is a complex task that takes place in our visual cortex and is affected by several factors, one of which is the continuation of contour elements. ${ }^{9}$ Good continuity in a visual scene is one of the six principles of grouping: a set of perceptual laws that defines the process of perceiving a single object as a coherent entity. Furthermore, some perceptual studies have shown that areas with high contrast and low pixel correlation within natural images attracts visual attention. ${ }^{10}$ Thus, the perception of an object, particularly within a cluttered environment, can be improved by a better continuity between contour elements and a higher contrast between the object and its surrounding. An edge enhancement technique, inspired by Cel-shading model, would satisfy both the continuation and the high contrast requirements.

\subsection{Volumetric Gradient-shading}

The illumination-reflection of MRA volumes is modeled after classic Flat-shading model where the reflected intensity is computed as a function of local surface normal. Surface normals, however, are explicitly defined in surfaces rather than volumes. To overcome this issue, the surface normal associated with a particular voxel is approximated using normalized gradient vectors. The intensity of each voxel is calculated by a combination of a gradient model and opacity value derived from the tissue type specified by the $2 \mathrm{D} \mathrm{TF}$. The choice of gradient shading is due to its low computational cost and therefore real-time volume visualization. Nevertheless, the resolution of MRA images is sufficiently high to compensate for the limitations (compared to Phong-shading or other smooth shading models) of the flat-shading model. Nevertheless, secular lighting was avoided in our gradient shading model as it would not increase the level of photo-realism nor the perception of depth. For instance, a shiny bone that is subcutaneous to other tissue types would not look natural in volume rendering.

\subsubsection{Volumetric Cel-shading}

Cel-shading is a non-photorealistic technique to create cartoon-like images, as if they were hand-drawn, using computer graphics. This model involves highlighting the contour lines with black ink to emphasize edges. Different methods can be used to create black ink contour lines, one of which takes advantage of a Z-buffer algorithm: rays are cast from the camera to a surface of interest, with each ray encountering a number of pixels along its path. Along each such path, the last salient voxels encountered are selected and their depth are recorded (Z-value). Each pixel is considered as a surface edge if the difference between their Z-value and their neighbour pixel's Z-value is greater than a certain threshold. In this study, however, a soft threshold function is used. Such a function, unlike standard or hard thresholding (all or nothing), has a sigmoid shape. Additionally, increasing the distance between two objects (i.e. relative depth) results into more ink around the edge. In volumetric images where the noise is not severe, the object's silhouette is located at discontinuities of the Z-buffer as viewed by the camera. ${ }^{11}$ If the volumetric data are opaque, the rays are terminated upon striking an opaque voxel. Early ray termination optimizes the rendering process, as the rays are not required to be traced all the way through the volume. ${ }^{12}$ However, when the object is translucent rather than opaque, rays will penetrate with sufficient depth through a translucent material such that opacity accumulates to a level where the ray stabilizes. ${ }^{13}$ In addition, the edge around an opaque object within a translucent cloud can be located using the Z-buffer method unless the ray is terminated before hitting the object.

\subsection{Experiment design}

\subsubsection{Rationale}

The primary focus of this study is to investigate whether applying certain shading models will enhance the perception of vessel connectivity and localization in MRA images. Clinically, volume rendering is often employed 


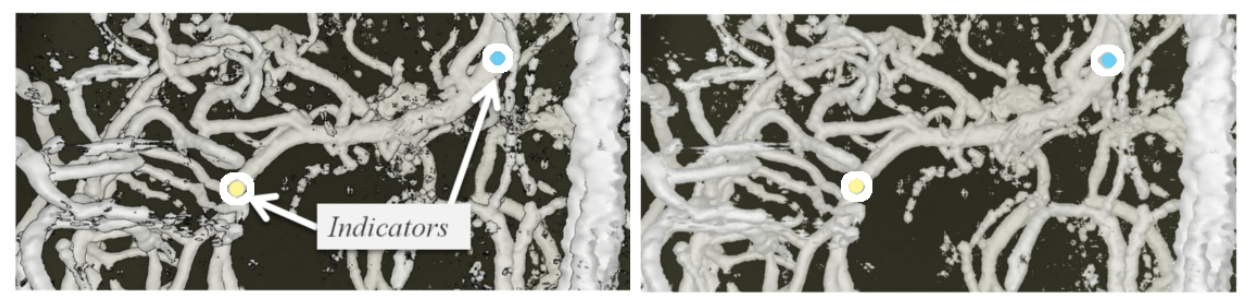

Figure 2. Participants are asked to decide on the connectivity relationship between certain vessels in stationary images of MRA data with (left) or without (right) Cel-shading

for visualizing different tissue structures within the same 3D scene. To better understand the anatomy of vascular tumours for instance, it is necessary to visualize the tumour and its surrounding vessels. In some cases, simultaneous visualization of the vessels in their context (i.e. the brain) is essential for better diagnosis or pre-operative planning. However, visualization of translucent tissue structures creates a foggy-looking cloud around the opaque objects. In direct volume rendering, however, rays penetrate into the cloud until the opacity accumulates to a level where they stabilize. For this reason, some of the structures within the cloud are not visualized, even when the transparency level of translucent area is set to the minimum possible value. On the other hand, contour enhancement using the Z-buffer is not sensitive to ray penetration but rather to the discontinuities of the Z-buffer. Therefore, applying Cel-shading contour enhancement will provide a very strong cue to the connectivity and local structure of opaque objects (e.g. blood vessels) by better delineating them within the cloudy areas (e.g. Fig. 1).

\subsubsection{Experiments}

Subjects $(\mathrm{N}=10)$ were presented with a series of stimuli $(\mathrm{n}=60)$ in a pseudo-random fashion. Stimuli consisted of stationary snapshots of volume-rendered MRA images with or without contour enhancement. During the experiment, participants were asked to decide on the connectivity relationship between certain vessels (whether or not the two vessels-of-interest are connected). Individuals were also asked to respond as quickly and accurately as possible while their responses and response times were recorded for analysis. Two colour-coded circles were placed on the surface of target vessels to indicate their position (Fig. 2). Furthermore, trials were categorized into three different categories of easy, medium, or difficult, depending on the level of cognitive effort needed to mentally connect the target vessels. The images were systematically placed in sequence such that the effects of learning and fatigue do not provide an advantage or disadvantage to either case. The actual connections between the target vessels were determined by examining the manually segmented vessels as well as exploring the 3D Volume-Rendered MRA image.

\section{RESULTS AND DISCUSSION}

\subsection{Perceptual Experiment}

Among ten volunteers who participated in this study, all of them but one experienced some improvement in their performance after being presented with enhanced stimuli. The subjects' success rates in terms of perceiving the connection between target vessels were improved by $8.4 \%$ on average after contour enhancement ( $\mu=75.6 \%$ vs. $84 \% ; p=0.02$, Cohen's d $=0.87$, Statistical power $=0.89$ ). Moreover, participants were not only more accurate but also faster in performing the task. The average response time were $2.3 \mathrm{sec}$ longer during the non-improved stimuli trials $(p=0.04)$ (Table 1$)$. In addition of paired T-tests, it's necessary to include more global test of significance as our results involve two simultaneously measured independent variables (accuracy and response time). For this end, calculated level of significance and the Hotelling's T-squared value using Hotelling's Tsquared test are equal to $p=0.01$ and 8.85 respectively. 


\begin{tabular}{|l|l|l|l|l|}
\hline Subject & \multicolumn{2}{|c|}{ Accuracy } & \multicolumn{2}{c|}{ Response Time } \\
\hline & edge-enhanced & Non-enhanced & edge-enhanced & Non-enhanced \\
\hline 1 & $90.00 \%$ & $70.00 \%$ & $10.7 \mathrm{sec}$ & $13.9 \mathrm{sec}$ \\
\hline 2 & $86.6 \%$ & $73.3 \%$ & $11 \mathrm{sec}$ & $15.5 \mathrm{sec}$ \\
\hline 3 & $93.3 \%$ & $83.3 \%$ & $12.4 \mathrm{sec}$ & $12.1 \mathrm{sec}$ \\
\hline 4 & $76.6 \%$ & $86.6 \%$ & $19.4 \mathrm{sec}$ & $21.6 \mathrm{sec}$ \\
\hline 5 & $60 \%$ & $60 \%$ & $6.2 \mathrm{sec}$ & $6.5 \mathrm{sec}$ \\
\hline 6 & $90 \%$ & $70 \%$ & $18 \mathrm{sec}$ & $27.8 \mathrm{sec}$ \\
\hline 7 & $76.6 \%$ & $73.3 \%$ & $7.9 \mathrm{sec}$ & $8.5 \mathrm{sec}$ \\
\hline 8 & $90 \%$ & $86.6 \%$ & $7.3 \mathrm{sec}$ & $7.2 \mathrm{sec}$ \\
\hline 9 & $93.3 \%$ & $83.3 \%$ & $7.8 \mathrm{sec}$ & $8.8 \mathrm{sec}$ \\
\hline 10 & $83.3 \%$ & $70 \%$ & $10.7 \mathrm{sec}$ & $12.1 \mathrm{sec}$ \\
\hline Mean & $84.00 \%$ & $75.60 \%$ & $11.17 \mathrm{sec}$ & $13.4 \mathrm{sec}$ \\
\hline
\end{tabular}

Table 1. Subject's accuracy and response time values
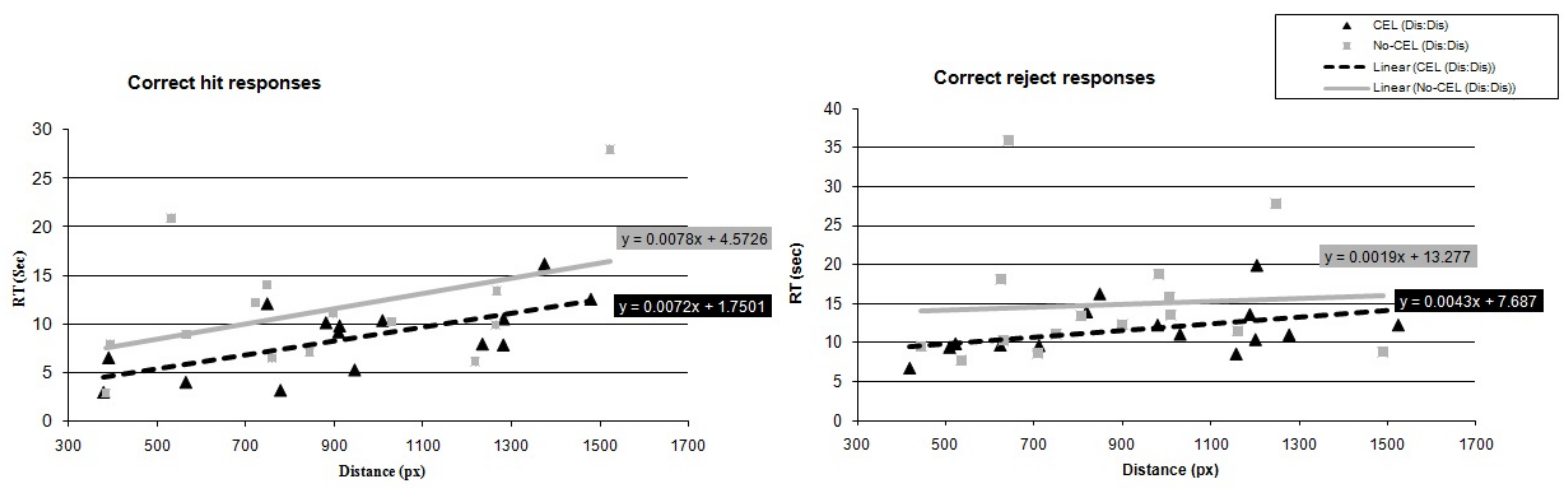

Figure 3. Reaction time vs. distance for correct 'hit' and correct 'reject' responses

Generally speaking, response time is proportional to the distance between the indicators placed on the target vessels, i.e. longer response times are supposedly coupled with greater distances between two indicators. Depending on whether or not the target vessels are connected, the correct responses can be categorized as either hit or reject. Nevertheless, the reject responses should be relatively less dependent on the distance as various number of visual cues could assist subjects to correctly identify the discontinuity without tracing the entire vessel. On the other hand, based on our hypothesis, trials with Cel-shaded images should require less time to respond. Thus, if our hypothesis is correct, then a) we should observe a proportional relationship between response time and distance; b) trials with contour enhancement, in general, present lower response time; and c) the best-fit lines to the correct hit dataset would have much steeper slope compare to the correct reject ones, mainly because longer response time is required to perform a task that involves tracing the entire vessel(s). Data presented in 3 indicates how these assumptions are fulfilled in our experiments.

In addition to our quantitative data, our participants agreed that it was less difficult to trace the vessels in the presence of contour enhancement. Hence, the overall result of this perceptual study shows how edge enhancement using a Cel-shading technique can improve the subjects' performance rate and reduce the time needed for perceiving the relationship between vasculatures in MRA data.

\subsection{Qualitative Feedback}

In addition of collecting quantitative data, an expert (neurosurgeon) was asked to perform standard pre-operative planning by exploring and examining an AVM patient data using our application and to provide us with his 
feedback. He was instructed to load a patient-specific MRA volume and specify a 2D transfer function using available widgets. He was also asked to deliberately change the amount of Cel-shading and Flat-shading darkness and enable (or disable) stereopsis. Using this interface, he found it easier to define the lesion of the nidus compared to standard pre-operative planning applications, particularly when both stereo and gradient-shading were enabled. The continuity of the veins and arteries, on the other hand, was easier to perceive when the Cel-shading model along with gradient-shading and stereo were applied. He believed that gradient-shading was helpful to provide an overall picture of the lesion whereas Cel-shading was beneficial in terms of following certain vasculatures. He believed that the best image was provided when gradient-shading and Cel-shading darkness were set to $75 \%$ and $50 \%$ of their maximum value, and the volume was rendered stereoscopically. Increasing the amount of Cel-shading darkness to greater than $50 \%$ was not desirable as it also emphasized the noise. Additionally, he found the interaction required to definine the 2D transfer function somewhat tedious. Nevertheless, he recognized the novelty and advantage of such application to plan AVM operations compared to conventional approaches and applications.

\section{FUTURE WORK AND CONCLUSION}

\subsection{Augmented Reality Platform}

Despite the dramatic changes in Minimally-Invasive Neurosurgery over the past few decades, the lack of reliable training and pre-operative planning schemes continues to be among the most challenging hurdles neurosurgeons have to face. ${ }^{14}$ Augmented Reality (AR) environments fuse information from a virtual space with real-time images of the physical world to provide a more intuitive way of exploring and examining patient-specific data. AR environments have not been widely embraced by the surgical community ${ }^{15}$ due to unsolved issues including poor interactivity, incorrect depth perception, suboptimal interface design, and prohibitive cost. We believe that we can address these issues by combining our knowledge of human visual and cognitive systems with state-of-the-art computer graphics techniques to reduce the ambiguity and improve performance in surgical applications. Thus, our future work will aims to develop and evaluate the use of an AR platform for training and planning complex neuro-surgical operations such as AVM. As part of the optimization process, our AR system would include user-dependent features to control and adjust visualization parameters such as contours, transparency, stereopsis and occlusion. To further increase the efficiency, irrelevant sections of data can be temporarily excluded in a semi-automatic fashion to reduce the high cognitive load demanded by surgical training and planning. Finally, to design a cost-effective platform, we make use of an off-the-shelf AR eyewear (Vuzix 920AR) equipped with twin stereoscopic cameras. In terms of evaluation, necessary user studies are conducted in close collaboration with neurosurgeons.

\subsection{Conclusion}

This study investigated whether intensifying the silhouette of certain structures in Volume-Rendered images, such as blood vessels in volumetric MRA images of a brain, can significantly facilitate the ability to perceive the relations between different tissue structures. In our experiments, we presented to our subjects a series of stimuli and asking them to perform a perceptual judgment task. Our results show that, in general, participants performed better within a shorter time during the trials with Cel-shaded stimuli. Accordingly, we believe that this technique can help radiologists and surgeons to better understand patients' vasculatures compared to traditional Volume-Rendering methods. Overall, our research introduces a novel concept that helps physicians to better explore and examine 3D Volume-Rendered MR Angiography images by emphasizing the edge around the

blood vessels. The proposed work has the potential to promote the quality of patient care by improving while lowering the cost of surgical training and conventional pre-operative planning platforms. 


\section{ACKNOWLEDGMENTS}

The authors would like to thank J. Moore, C. Wedlake, and Dr. E. Cheng for valuable discussions and technical support. This project was supported the National Science and Engineering Research Council of Canada (NSERC \#RE3146A04), the Canadian Foundation for Innovation (CFI \#20994), and in part by the Natural Sciences and Engineering Research Council (NSERC) of Canada through a Collaborative Research and Training Experience (CREATE) program grant (CAMI \#371322-2009) in Computer-Assisted Medical Interventions. Graduate student funding for Kamyar Abhari was provided by scholarships from the National Science and Engineering Research Council of Canada and by the University of Western Ontario.

\section{REFERENCES}

[1] Larijani, L., [The virtual reality primer], McGraw-Hill (1994).

[2] NIH., "NINDS arteriovenous malformation information page," (2009).

[3] Weiler, F., Rieder, C., David, C., Wald, C., and Hahn, H., "AVM-Explorer: Multi-volume visualization of vascular structures for planning of cerebral AVM surgery," in [Eurographics], (2011).

[4] Ehricke, H., Schad, L., Gademann, G., Wowra, B., Engenhart, R., and Lorenz, W., "Use of MR Angiography for stereotactic planning," Journal of Computer Assisted Tomography 35, 585-591 (1992).

[5] Drebin, R., Carpenter, L., and Hanrahan, P., "Volume Rendering," SIGGRAPH Comput. Graph. 22, 65-74 (June 1988).

[6] Fishman, E., Ney, D., Heath, D., Corl, F., Horton, K., and Johnson, P., "Volume rendering versus maximum intensity projection in CT Angiography: what works best, when, and why.," Radiographics 26, 905-922 (2006).

[7] Wallis, J., Miller, T., Lerner, C., and Kleerup, E., "Three-dimensional display in nuclear medicine," IEEE Trans Med Imaging 8, 297-303 (1989).

[8] Zhang, Q., Eagleson, R., and Peters, T. M., "Dynamic real-time 4D cardiac MDCT image display using GPU-accelerated volume rendering," Computerized medical imaging and graphics 33, 461-476 (2009).

[9] Miikkulainen, R., Bednar, J., Choe, Y., and Sirosh, J., [Computational maps in the visual cortex], Springer Science+Business Media, Inc (2005).

[10] Reinagel, P. and Zador, A., "Natural scene statistics at the centre of gaze," Network 10, 341-350 (1999).

[11] Decaudin, P., "Cartoon looking rendering of 3D scenes," Research Report 2919, INRIA (June 1996).

[12] Turner, W., "An improved illumination model for shaded display," SIGGRAPH Comput. Graph. 13, 14(1979).

[13] Levoy, M., "Efficient ray tracing of volume data," ACM Trans. Graph. 9, 245-261 (1990).

[14] Cosman, P., Hemli, J., Ellis, A., and Hugh, T., "Learning the surgical craft: A review of skills training," ANZ J. of Surg 77, 838-845 (2007).

[15] Sielhorst, T., Feuerstein, and Navab, N., "Advanced medical displays: A lit. review of augmented reality," $J$ of Display Tech 44, 451-467 (2008). 